\title{
e-Phaïstos
}

e-Phaïstos

Revue d'histoire des techniques / Journal of the history

of technology

II-2 | 2013

Les sources de l'histoire des techniques (2)

\section{Les relations entre pensée technique et pensée économique}

Entretien avec Benjamin Ravier-Mazzocco

The Relationship between Technical and Economic Thinking. Interview with

Francois Vatin by Benjamin Ravier-Mazzocco

\section{François Vatin}

\section{(2) OpenEdition}

Journals

\section{Édition électronique}

URL : https://journals.openedition.org/ephaistos/7361

DOI : 10.4000/ephaistos.7361

ISSN : 2552-0741

\section{Éditeur}

IHMC - Institut d'histoire moderne et contemporaine (UMR 8066)

\section{Édition imprimée}

Date de publication : 15 décembre 2013

Pagination : 86-94

ISSN : 2262-7340

Référence électronique

François Vatin, «Les relations entre pensée technique et pensée économique », e-Phaïstos [En ligne], II-2 | 2013, mis en ligne le 12 décembre 2019, consulté le 16 février 2022. URL : http:// journals.openedition.org/ephaistos/7361 ; DOI : https://doi.org/10.4000/ephaistos.7361 


\title{
Entretien avec François Vatin Les relations entre pensée technique et pensée économique
}

\author{
Par Benjamin Ravier-Mazzocco
}

\section{Cursus académique}

1978 : Maîtrise de Sciences Economiques, Faculté des Sciences Economique, Aix-en-Provence.

1981: Reçu docteur d'étude interdisciplinaire du changement social et des mouvements sociaux, option économie, EHESS, Aix-Paris, avril, 1981. Titre de la thèse : "L'économie des flux, essai de généalogie et de synthèse de l'organisation industrielle des procès de production continus, » sous la direction de Jean-Paul de Gaudemar, Professeur de Sciences économiques.

1979-1983: Chercheur contractuel au laboratoire de conjoncture et prospective (Var), allocataire de recherche DGRST au titre de l'EHESS (Aix), puis chargé d'études et de missions sur l'emploi à la direction départementale de la Jeunesse et des sports des Bouches-du-Rhône.

1983-1988 : Assistant de sciences économiques, université de Rennes 2.

1988-1992 : Maître de conférences en sciences économiques, université de Rennes 2.

1991 : Habilitation à diriger des recherches en Sociologie, Université de Paris X-Nanterre. Titre du mémoire : "Théorie et pratiques du travail et de la production ».

Depuis octobre 1992 : Professeur de Sociologie à l'Université de Paris X-Nanterre

Depuis 2010 : Directeur du site de Nanterre de l'IDHE (Institutions et dynamiques historiques de l'économie)

\section{Bibliographie sélective}

La fluidité industrielle, essai sur la théorie de la production et le devenir du travail, préface de $\mathrm{P}$. Naville, Paris, Méridiens-Klincksieck, 1987

Le travail, économie et physique (1780-1830), Paris, PUF, 1993, traduit en grec en 1995

Le lait et la raison marchande, essais de sociologie économique, PUR, Rennes, 1996.

Édition et annotation de la thèse de Bernard Grall (1962-1997), Economie des forces et production d'utilité. La pensée gestionnaire des ingénieurs des ponts (1831-1891), PUR, Rennes, 2004.

Traité de sociologie du travail, Toulouse, Octares, 2003 (en collaboration avec Thierry Pillon), seconde édition actualisée, 2007.

Travail, sciences et société, essais d'épistémologie et de sociologie du travail, Bruxelles, Presses de l'Université de Bruxelles, 1999, traduit en portugais en 2002 et en espagnol en 2004.

Évaluer et valoriser. Une sociologie économique de la mesure, Toulouse, Presses universitaires du Mirail, 2009, seconde édition enrichie, 2013.

Co-directeur avec Philippe STEINER, Traité de sociologie économique, Paris, PUF-Quadrige, 2009, seconde édition enrichie, 2013.

L'espérance-monde. Essais sur l'idée de progrès à l'heure de la mondialisation, Paris, Albin Michel, coll. Bibliothèque idées, 2012. 
$\boldsymbol{e}$-Phaïstos : Vous avez été l'un des pionniers dans le rapprochement entre des concepts de physique mécanique et des concepts d'économie, qu'est-ce qui vous a amené à ces recherches ?

François Vatin : Je suis économiste de formation, et me suis intéressé à l'organisation industrielle, en particulier aux débats autour de l'organisation "scientifique » du travail au début du $\mathrm{XX}^{\mathrm{e}}$ siècle, à la place qu'occupait la pensée d'ingénieur sur ces questions du travail, avec, notamment, la figure emblématique de Taylor. J'avais fait ma thèse sur l'organisation industrielle dans les industries à processus de production en continu, tels le raffinage pétrolier ou la pétrochimie.

La question qui m'oriente depuis le début de mes travaux, c'est celle de l'efficacité industrielle, de la production de la valeur. Question cruciale dans ce type de processus productif où la production se réalise en continu et où il est très difficile de penser le processus de valorisation, en tous cas selon un schéma classique de sommation de travail, qui est encore celui de Taylor ${ }^{1}$. Or, à la fin des années 1970début des années 1980, il y avait toute une série de travaux en cours qui portaient sur l'analogie entre l'économie et la thermodynamique, sujet en lien avec les réflexions sur le risque d'épuisement des gisements de pétrole (club de Rome etc.). Dans ces travaux, on voyait à l'œuvre des analogies entre énergie et valeur, pensées à un niveau épistémique. J'imaginais donc là une voie de réponse à mes questions.

C'est en m'intéressant à ce sujet que j'ai découvert le corpus de la mécanique industrielle, c'est-àdire le discours des ingénieurs des années 18201830 qui élaborent le concept de "travail mécanique ». Je me suis aperçu que, dans leurs textes, la référence à l'économie politique était omniprésente. Il ne s'agissait pas d'une construction analogique souterraine que l'épistémologue pouvait mettre à jour à la manière des strates épistémiques de Foucault. De façon explicite, pour ces ingénieurs, il fallait introduire la pensée économique en mécanique, « soumettre la mécanique à l'économie poli- tique » pour reprendre l'expression de l'ingénieur des Mines Claude Burdin en $1815^{2}$. Voilà comment j'ai été amené à croiser économie et physique en me posant une question toute simple : pourquoi parlet-on de «travail » en mécanique 3 ?

Le travail mécanique est appelé comme tel en référence au travail humain dans une double analogie : comme l'homme, la machine "produit », mais aussi, comme l'homme, elle dépense pour produire. Cette double dimension du travail, clairement posée par Charles Coulomb pour le travail humain dès 1799 dans son texte connu sous le titre Mémoire sur la force des hommes ${ }^{4}$, conduit au concept de rendement, c'est-à-dire à la construction d'un rapport entre un travail produit (travail utile) et un travail dépensé (travail total) qui est au fondement de la mécanique industrielle; le projet est de concevoir des machines au rendement maximal praticable, soit de minimiser la perte.

$\boldsymbol{e}$-Phaïstos : Est-ce qu'on peut revenir en détail sur ce premier corpus qui vous a amené à travailler sur le sujet et de là, expliquer cette première généalogie des années 1820,1830 ?

F.V. : J'avais eu la chance de commencer par la lecture d'un opuscule de Stéphane Flachat 5 , qui consistait en une compilation des travaux de l'époque (extraits de Navier, Coriolis, Christian, Poncelet, Dupin, etc.), ce qui m'a permis de remonter immédiatement aux textes principaux. Notamment, et c'est, je pense, le point crucial qui m'a amené à produire un travail original, je suis ainsi « tombé » sur un auteur souvent mis de côté dans l'histoire « internaliste » de la construction du concept de travail mécanique: Charles Coulomb. Quand on lit la littérature des années 1820-1830 sur le concept de "travail mécanique», le Mémoire sur la force des hommes de Coulomb apparaît comme la matrice fondamentale de la mécanique industrielle. Elle inscrit cette discipline dans une optique économique qui fait écho aux débats de l'économie politique classique sur la «valeur travail », puisque son enjeu est l'optimisation de l'emploi des hommes 
au travail. La transmission entre Coulomb et la mécanique industrielle se fait notamment via Navier, qui, dans une des additions qu'il fournit au texte de Bélidor dans sa réédition de l'Architecture hydraulique de cet auteur ${ }^{6}$, suit de très près le mémoire de Coulomb.

Ce passage par Coulomb invite à aborder un autre aspect de la question : l'étude mécanique du travail humain. Ce qui nous ramène, via la physiologie, aux débats de la fin du XIX ${ }^{\mathrm{e}}$ siècle et du début du $\mathrm{XX}^{\mathrm{e}}$ siècle sur l'organisation scientifique du travail évoqués plus haut. On a donc deux questions analogues qui se croisent: le rendement des machines et celui des hommes. Ce qui me donnait cette clé de lecture, c'était bien le fait que je m'étais intéressé précédemment aux débats sur le travail à l'époque de Taylor, et, notamment, à un auteur comme Jules Amar. Ce physiologiste français avait soutenu en 1909 une thèse intitulée Le rendement de la machine humaine ${ }^{7}$. C'est l'expression la plus nette d'une tentative de saisie exclusivement mécaniste, énergétiste du travail humain. Cette histoire a été racontée notamment par Anson Rabinbach dans Human Motor ${ }^{8}$, qui traite de la physiologie du travail de la fin XIXe-début XXe comme une application au travail humain du modèle énergétiste. $\mathrm{Ce}$ qui n'est pas faux, mais tronqué : on ne comprend rien en effet si on ne voit pas que ce n'est qu'un "prêté pour un rendu ». Si on applique la mesure physique du « travail » au travail humain à la fin du XIX $^{\mathrm{e}}$ siècle, c'est bien parce que, préalablement, les mécaniciens avaient, au début du XIX ${ }^{\mathrm{e}}$ siècle, opéré à l'inverse, la transposition de l'homme à la machine. Par ma démarche, qui prenait Coulomb comme point de départ, je modifiais donc, en même temps, une certaine histoire de la mécanique, et une certaine histoire de la physiologie du travail. Je mettais en évidence un fonctionnement sinusoïdal de la circulation des concepts entre l'homme et la machine, qui domine toute cette " science croisée » du travail, depuis le début du XVIII ${ }^{e}$ siècle avec Amontons, jusqu'au début du XXe siècle avec Amar.
$\boldsymbol{e}$-Phaïstos : Je voudrais revenir sur les propriétés du travail qui font qu'on a pu le considérer comme une monnaie mécanique, qu'est-ce qui fait qu'au départ, ces premières transpositions du travail humain vers la mécanique ont pu fonctionner comme mesure économique ?

F.V. : La question qui est posée depuis Amontons ${ }^{9}$ est : comment mesurer l'utilité économique d'une machine? Amontons répondait : à la capacité qu'a cette machine de remplacer les hommes (ou les chevaux). Il s'agit donc de mesurer l'activité de la machine en équivalent-hommes (ou équivalentchevaux).

Or, parallèlement, le travail humain va de plus en plus s'exprimer dans un coût monétaire avec l'émergence de ce qu'on peut appeler le salariat, pour aller vite. Et cela s'incarne effectivement chez Coulomb, auteur paradoxal, puisqu'il raconte luimême que s'il s'est intéressé à la mesure de la capacité de travail des hommes, c'est à l'occasion de ses travaux de construction du fort de France à la Martinique dans les années 1760. Or, à la Martinique, à cette époque-là, il n'a pu manquer d'avoir affaire à du travail forcé. Pourtant, le modèle que développe Coulomb est profondément libéral, puisque ce qui lui permet de boucler ses équations, c'est un principe d'égalité marchande. Il considère, un peu comme Smith, que deux travaux payés le prix « ordinaire » d'une journée de travail contiennent une quantité égale de fatigue. C'est donc par le marché que peuvent s'équilibrer les travaux qu'il cherche à mesurer mécaniquement.

Il y a donc deux jeux de mise en équivalence qui s'opèrent: un premier jeu de mise en équivalence de l'activité de la machine avec celle de l'homme et un second jeu de mise en équivalence de la force mécanique et de la monnaie. C'est par le jeu de cette double équivalence qu'est pensée l'économie de la machine au début du XIX ${ }^{\mathrm{e}}$ siècle. Sans revenir sur la « querelle des forces vives ${ }^{10}$ qui agite les physiciens tout au long des XVII e et XVIII e siècle, il faut rappeler qu'elle est tranchée au début du XIX ${ }^{\mathrm{e}}$ siècle par les mécaniciens par l'invocation de cette for- 
mule, attribuée à Joseph Montgolfier : "La force vive est celle qui se paie $»^{11}$. Finalement, c'est en monnaie que ça se joue, et nous avons là une articulation très étroite, à un niveau épistémique, des représentations de la physique et de l'économie au moment même où se développent les relations marchandes ${ }^{12}$.

Sur cette base, les mécaniciens découvrent, dans les années 1820-1830, les propriétés métrologiques formelles du travail mécanique, lequel est une grandeur scalaire, donc additive. Cette propriété formelle, similaire à celles de la monnaie pour les économistes, permet de faire du travail mécanique l'équivalent général de tous les échanges mécaniques. Chose que Cournot formule de façon magistrale, dans un ajout à un traité de mécanique anglais, qu'il traduit en 1834 : " La force vive est ce qui se paie dans une machine destinée à la transmission et la transformation du mouvement, en ce sens qu'on paie la machine selon le rapport de la force vive qu'elle recueille à la force vive qu'elle transmet aux pièces directement chargées de l'effet utile [le rendement, FV]. En un mot, la force vive est de tous les effets dynamiques le seul qui se conserve, s'emmagasine, se transmet, s'échange, se fractionne et il faudrait bien peu être initié dans la science économique pour ne pas voir que qu'à ce titre seul, la force devait devenir l'étalon dynamique, lors même que le travail le plus actuel des machines ne serait pas directement mesuré par la force vive. C'est en vertu de propriétés analogues que les métaux précieux servent d'étalons à toutes les valeurs commerciales, sans être, à beaucoup près, les denrées dont la consommation directe est la plus fréquente et la plus impérieusement réclamée par nos besoins. »13

Comme on le voit, le rapprochement entre économie et physique, je ne le fais pas a posteriori, de l'extérieur. Il figure explicitement dans les textes. Pourquoi prendre le travail comme mesure de l'activité des machines ? Comme Cournot le montre bien, c'est en raison des propriétés formelles de cette grandeur, de son additivité. Tout comme un objet à 10 francs et un objet à 5 francs valent ensemble 15 francs, on peut additionner le travail réalisé par plusieurs machines. C'est là une condition fondamentale pour développer un calcul de l'effet des machines. Cela n'empêchera pas pour autant que la pertinence de cette grandeur comme mesure générale des échanges mécaniques soit discutée.

$\boldsymbol{e}$-Phaïstos : N'y a-t-il pas une critique de cette «monnaie mécanique », par exemple avec une distinction similaire à celle que Marx fait entre la valeur utile et la valeur d'échange? Ne peut-on pas dire qu'une opération se mesure aussi à ce qu'elle réalise en dehors du travail qu'elle fournit ?

F.V. : Le fond du modèle, c'est que toute opération peut-être incarnée par une dépense de travail, puisqu'on pourra toujours remplacer l'effet de l'outil par un poids élevé à une certaine hauteur. Ce qui échappe à ce modèle, c'est, bien évidemment, la «qualité » de l'opération, mais il faut rappeler qu'on est ici dans une représentation de la production de type grossière. La référence, c'est le moulin, utilisé pour moudre le grain ou pour alimenter les travaux de force d'une forge, par exemple.

Le point sur lequel les ingénieurs vont très vite buter en revanche, c'est la question du temps. La force, mais aussi la faiblesse, de la mesure mécanique en travail, c'est la non-prise en considération du temps, ou plus exactement de la vitesse à laquelle l'opération est effectuée. Cette question du temps rencontre là encore des interrogations de l'économie politique, à savoir les concepts de capital et de taux d'intérêt. Pour parer aux questions de vitesse, la solution la plus souvent avancée est la possibilité d'installer plusieurs machines en parallèles, pour obtenir un produit plus abondant dans le même temps. Mais on ignore alors le coût de la machine (le coût d'immobilisation du capital). La question est exactement la même que celle que posait Ricardo à propos de la valeur travail, à savoir : est-ce que le travail d'un homme pendant cent jours est équivalent à celui de cent hommes pendant un 
jour ? Ce sont bien cent journées de travail dans les deux cas, mais, dans le premier cas, le produit est disponible au bout de cent jours, et, dans l'autre, au bout d'un jour. Ce n'est donc pas équivalent si on considère le prix du temps, ce que les économistes appelleront la «préférence pour le présent », qui détermine le taux d'intérêt.

Pour la mécanique industrielle, la démarche est la même : confronté à cet instrument de mesure (le « travail »), qui élimine le temps pour pouvoir additionner, répartir etc., elle en vient à poser la question des frais d'installation (du capital donc), c'està-dire du coût de la machine elle-même, qui lui, ne peut pas être mesuré en travail.

Sur cette question aussi, le croisement entre économie et physique est explicite dans les textes des ingénieurs. Il ne s'agit pas d'une métaphore. Les ingénieurs sont confrontés aux mêmes problèmes analytiques que les économistes. Prenons Coriolis par exemple: " La distinction entre le temps et le travail dans l'évaluation des moteurs est tout à fait semblable à celle qui doit se faire dans l'achat de certaines matières premières, entre la quantité qu'on achète et le temps qui sera employé à la livraison. [N'oublions pas qu'il s'agit d'ingénieurs des Ponts et Chaussées, habitués à acheter des matières premières en grandes quantité et à tenir compte des délais de livraison, F.V.] Quoiqu'il soit souvent très utile que la fourniture d'une marchandise qui s'effectue peu à peu, à tant par jour, soit terminée dans huit jours, au lieu de l'être dans un mois. Cependant, cela n'empêche pas que la quantité de cette marchandise ne forme toujours l'élément principal du marché, et celui qu'on ne pourrait omettre d'énoncer dans le contrat. »14

Ce que Coriolis nous dit ici, c'est que, même dans ces cas là, la marchandise, la matière première, est le cœur du marché. Il se justifie ainsi du choix d'une mesure en travail alors même qu'il est conscient du caractère biaisé de la mesure: de même que, dans l'économie marchande, on va prendre comme mesure la quantité de marchandises en sachant qu'on ignore ainsi toute une série de dimensions du problème, de même, en mécanique, on prend en considération le travail, alors même qu'on a conscience que ça ne prend pas en considération la totalité des données de l'économie de la machine. On hiérarchise donc les données du problème.

La question qui se pose à partir de là, et j'en viens à votre question précise, c'est celle de la qualité. Reprenons Coriolis : «Il y a des cas où en cherchant à économiser un style de travail, on pourrait ne pas obtenir les mêmes produits sans que cela paraisse au même abord. Ainsi, en laminant du fer, on n'obtient pas une aussi bonne qualité qu'en le battant. C'est au fabricant à examiner jusqu'à quel point on peut faire ainsi des économies de travail au dépend de la qualité des produits. ». C'est là le cœur du problème, car ces utilités ne peuvent être mesurées, calculées, et modifiées à convenance, en restant dans la seule métrologie du travail.

$\boldsymbol{e}$-Phaïstos : La citation est intéressante, qui explique que c'est au fabriquant d'estimer, « d'examiner », la perte de qualité acceptable.

F.V. : Bien sûr, l'objectif de la mécanique industrielle qui se met en place, c'est de fournir des normes d'efficacité pour l'organisation de l'industrie, avec cette idée majeure que la priorité est d'économiser le « travail »; c'est-à-dire de construire et de régler les machines afin qu'elles aient un rendement maximum, que les pertes de travail mécaniques soient les plus faibles possible. La question est alors : dans quelle mesure a-t-on ainsi défini une norme d'efficacité industrielle pertinente? Il est donc logique que ce soit ensuite à l'industriel de faire la part des choses, en y ajoutant les dimensions non prises en compte par ce modèle.

e-Phaïstos : Cette question du temps dans le fonctionnement des machines avait déjà fait l'objet de plusieurs remarques, plutôt acerbes, au cours de la période moderne. Revenant au levier d'Aristote, la statique, qui ramenait aussi tous ces calculs à l'élévation d'un poids, ne prenait pas en compte le 
temps. Or très vite, alors même que le frottement ne faisait pas l'objet d'études approfondies, les savants critiquent la multiplication des roues dentées, censées démultiplier la force motrice, mais sans prendre en compte le temps. Y a-t-il ici une continuité ou une rupture avec ce corpus antérieur ? Sur quelle science des machines s'appuient ces auteurs?

F.V. : Ce terrain est déjà moins le mien, mais je pense qu'il y a une assez grande continuité, parce que la mécanique industrielle prend appui sur les grands traités du XVIII siècle. Les apports importants de Navier, par exemple, sont établis à l'occasion de la rédaction de ses notes pour la réédition du traité de Bélidor, qui paraît en 1819. C'est donc qu'il paraît encore utile à cette période de mettre à disposition des ingénieurs ce texte classique du XVIII ${ }^{e}$ siècle. Je ne vois donc pas de rupture profonde.

e-Phaïstos : Dans cette continuité, est-ce que les textes de cette période font des références explicites à la technologie, telle que la définit Beckmann, ou à des textes antérieurs?

F.V. : Il y a déjà un lien direct, dans la personne de Christian, que j'ai cité tout à l'heure. Considéré dans l'article de référence de Guillerme et Sebestik ${ }^{15}$ comme un des fondateurs de la technologie [qu'il appelle technonomie], Christian est aussi l'auteur d'un traité de mécanique industrielle. Sur le plan historiographique, il y a donc bien une certaine continuité.

Cependant, plus profondément, et sur un plan épistémologique, votre question m'amène à préciser ce qui m'anime dans ces recherches : ma conviction que la notion même d'efficacité technique est intrinsèquement économique, à l'inverse de ce que laisse penser l'opposition, installée dans la seconde moitié du $\mathrm{XX}^{\mathrm{e}}$ siècle, entre rationalité économique et rationalité technique. Max Weber s'est essayé à poser cette distinction, dans Économie et société, sans y parvenir toutefois à mon jugement ${ }^{16}$. Mais cette division entre deux types de rationalité est finalement assez récente. Le Traité de Jacques Le- sourne, par exemple, insiste sur la distinction, en traçant, par l'introduction du prix, une ligne de partage entre l'ingénieur d'un côté et l'économiste de l'autre ${ }^{17}$. L'idée est que le technicien proposerait une batterie de solutions techniquement définies, et que, dans un second temps, l'économiste introduirait le prix dans le système pour opérer le choix dans un panel de techniques. Or, l'histoire que j'ai racontée plus haut contredit cette idée. Elle montre que l'instrument qu'utilisent les ingénieurs tout au long du $\mathrm{XIX}^{\mathrm{e}}$ siècle, à savoir le travail mécanique, est bien pensé comme économique. C'est une mesure pensée en relation avec cette autre mesure qu'est la monnaie : il y a donc une continuité totale entre une mesure et une optimisation en « travail » et une mesure et une optimisation en monnaie. C'est ce qu'a très bien montré Bernard Grall dans Économie de forces et production d'utilités, en étudiant la pensée des ingénieurs des Ponts ${ }^{18}$. Le passage de l'économie de la route (sans monnaie, car la route est gratuite en France) à l'économie du chemin de fer (monétaire) s'opère sans solution de continuité. Ce sont les mêmes modèles où la monnaie vient remplacer le travail mécanique comme unité de compte.

La question technique est pour moi centrale à travers cette idée que toute norme d'efficience technique est intrinsèquement économique et que la monnaie n'est qu'un instrument parmi d'autres, dans ces métrologies de la production. J'ai fait des travaux équivalents, par exemple sur l'agronomie.

e-Phaïstos : Par exemple?

F.V.: Sur l'agronomie il y a un texte que je trouve assez drôle, où un agronome cherche à déterminer ce qu'il faut faire pour valoriser au mieux l'élevage laitier ${ }^{19}$ : élever le veau sous la mère? Vendre du beurre, du lait, du fromage, etc. ? Pour normaliser ses calculs, il a besoin d'un équivalent général; il pose ainsi un "équivalent fumier ». Au $\mathrm{XIX}^{\mathrm{e}}$ siècle, vous trouvez exactement le même type de choses dans des corps de pensée très variés. 
$\boldsymbol{e}$-Phaïstos : Vous développez là l'aspect énergétique de la technologie, notamment appliquée à la mécanique. Au XVIII ${ }^{\mathrm{e}}$ siècle, la technologie est aussi un mode de description des métiers, de leurs principes, qui amène Beckmann à penser des classes d'opérations dans son second ouvrage de 1806. Voit-on au XIX ${ }^{\mathrm{e}}$ siècle des réflexions similaires sur cette façon de penser les métiers?

F.V.: L'histoire de la cinématique au XIX ${ }^{\mathrm{e}}$ siècle, de Babbage à Reuleaux, en passant par Willis et Laboulaye, est parallèle à celle de l'énergétique du travail. Mais je ne me suis jamais penché de façon un peu approfondie sur la question. Je me suis un peu intéressé à la cinématique humaine à la fin du XIX ${ }^{e}$ siècle-début XXe chez un auteur comme Armand Imbert ${ }^{20}$, car elle pose des questions immédiatement énergétiques : la structure cinématique de l'organisme humain est une donnée qui impose des contraintes énergétiques, alors que la structure cinématique d'une machine est l'objet de la construction imaginative du mécanicien.

Pour en revenir à la littérature du début du XIX ${ }^{\mathrm{e}}$ siècle, vous avez un auteur qui est intéressant sur ce point, c'est Charles Dupin, qui applique directement le principe d'économie du travail aux gestes humains. Voyons son commentaire d'un de ses graphiques représentant un travail statique: "Regardons une poissarde, par exemple, dont l'étalage tenu par les bretelles est horizontalement suspendu devant elle. Elle se tient très droite, porte le haut du corps et la tête en arrière. Souvent, appuyant les points sur les hanches, elle porte pareillement ses coudes en arrière. Habitude qu'elle ne prend pas d'ordinaire pour se donner une attitude quinteuse et menaçante, mais pour porter sans fatigue le centre de gravité du corps et des bras aussi en arrière que possible afin de faire contrepoids à son étal ${ }^{21}$. Cela s'inscrit chez Dupin dans une conception à la fois industrielle et sociale d'une économie généralisée, qui est au cœur de son cours de mécanique et de géométrie appliquées aux Arts au Conservatoire. Le but est de montrer que l'enseignement de la géométrie et de la mécanique permet d'améliorer l'effort productif. Il commence alors par les «techniques du corps » pour parler comme Marcel Mauss ${ }^{22}$. Il y a donc un continuum complet pour lui entre ce qui pourra être apporté par la géométrie et la mécanique, pour l'amélioration des machines et pour l'utilisation rationnelle du corps humain. Il s'agit toujours de trouver la configuration qui fournit le maximum de produit avec le minimum de dépense. À la base de toute cette réflexion, nous retrouvons une nouvelle fois le mémoire de Coulomb de 1799.

D'ailleurs, ce passage montre bien que, tout en essayant de montrer que tous les métiers peuvent profiter d'une meilleure connaissance de la géométrie de la mécanique, Dupin emprunte à Coulomb l'idée que la pratique, la routine, conduit à l'obtention d'un optimum que la Science peut retrouver. La poissarde a trouvé les bons gestes, la bonne posture ici, du fait d'une transmission par l'expérience, la sienne et celle de la tradition; c'est dans le même esprit que Coulomb étudiait le travail des hommes. De même, dans son mémoire sur la construction des moulins à vent, il conclut ses calculs en affirmant que les artisans avaient, par essais-erreurs, trouvé la bonne forme des ailes. Coulomb présuppose toujours que l'action du travailleur est marquée par un principe de rationalité, tant économique que technique. Dans un passage très drôle de son étude du portage vertical, il aboutit par le calcul à la détermination d'une charge optimale qui est un peu moins élevée que celle que les porteurs portent réellement. Il n'accuse pas du tout en conséquence les ouvriers de routine ou d'irrationalité, il ne dit pas qu'ils se fatiguent inutilement, mais il fait rentrer de nouveau le marché en ligne de compte : les porteurs étant en concurrence sur le marché, ils ont intérêt à paraître un peu plus forts qu'ils ne le sont vraiment. Nous voyons qu'il n'y a pas de représentation d'un travail indigène sous-productif que la science viendrait régler, comme chez Taylor, mais au contraire cette idée qui traverse le XVIIIe siècle, qu'il faut aller apprendre auprès des artisans. 
$\boldsymbol{e}$-Phaïstos : Nous venons de voir la richesse d'une recherche sur l'aspect économique de certains concepts issus de la littérature des ingénieurs des $\mathrm{XVIII}^{\mathrm{e}}$ et surtout $\mathrm{XIX}^{\mathrm{e}}$ siècle ; une recherche qui aide les historiens des ingénieurs à éclairer les invariants et les évolutions de leur pensée technique. Aussi, pour conclure, pourriez-vous nous dire comment vous voyez l'intérêt de ces recherches pour l'économie ou la sociologie du travail en général?

F.V.: En m'intéressant à ces questions d'épistémologie et d'histoire des sciences, je n'ai en fait jamais abandonné une perspective d'économiste et de sociologue du travail. Les textes m'y ont aidé, car le travail vivant, les formes d'organisation industrielle et les questions sociales qui en découlent, sont présentes en permanence dans cette littérature d'ingénieurs et de physiologistes, qui s'appuie, parallèlement, sur la mécanique, la physique ou la biologie. La question majeure est celle de la mesure de l'efficacité, c'est-àdire de la rationalité économique. C'est une question qui, d'un côté, impose la rigueur du calcul et de la métrologie «scientifique », mais qui, de l'autre, ne peut pas être dissociée de l'univers flou et chatoyant des «valeurs » sociales. Qu'est-ce qui « coûte »? Qu'est-ce qui « rapporte »? L'homme, dans son rapport technique au monde, s'affronte aux «forces naturelles » et, de cette confrontation productive, se dégagent des métrologies qui ont rapport avec la matérialité du monde, y compris quand les forces dont il est question sont celles de son propre organisme. Mais, d'un autre côté, ces métrologies ne peuvent se penser en dehors des normes individuelles et collectives $\mathrm{du}$ 《bon », du « bien », de l' « efficace », de l'adapté, du «profitable » en somme, et, de l'autre, du fatigant, du pénalisant, du « coûteux » en toute généralité. C'est ainsi la question fondamentale de l'économique qui se donne à lire de façon particulièrement pure dans la littérature des ingénieurs. Le concept apparemment si simple de « rendement mécanique » (travail utile produit sur travail total dépensé) est une forme archétypale pour penser la question de la «rationalité économique». Elle invite en effet à retrouver l'étymologie même de «rationalité »: le ratio, c'est-à-dire le rapport.

${ }^{1}$ VATIN François, La fluidité industrielle. Essai sur la théorie de la production et le devenir du travail, Paris, MéridiensKlincksieck, 1987.

2 BURDIN Claude, « Considération générales sur les machines en mouvement », Journal des Mines, $\mathrm{n}^{\circ}$ 221, 1815.

3 VATIN François, Le travail, économie et physique, 1780-1830, Paris, PUF, 1993.

4 Le titre exact du mémoire lu initialement à l'Académie en 1775 et augmenté jusqu'en 1798 est : "Résultats de plusieurs expériences destinées à déterminer la quantité d'action que les hommes peuvent fournir par leur travail journalier, suivant la manière dont ils emploient leurs forces", Mémoires de l'Académie des sciences de Paris, 1799 repris dans Théorie des machines simples, Paris, Bachelier, 1821.

5 FLACHAT Stéphane, Traité élémentaire de mécanique industrielle : résumé des traités de Christian, Poncelet..., Paris, chez L. Tenré et H. Dupuy, 1835.

6 BELIDOR (de) Bernard-Forest et NAVIER Claude-LouisMarie-Henri, Architecture hydraulique : ou l'art de conduire, d'élever, et de ménager les eaux pour les différents besoins de la vie, nouvelle édition, Paris, Firmin Didot, 1819.

7 AMAR Jules, Le rendement de la machine humaine, Paris, Baillière, 1909.

8 RABINBACH Anson, The human motor : energy, fatigue, and the origins of modernity, Berkeley et Los Angeles, University of California Press, 1992; traduction française : Le moteur humain : l'énergie, la fatigue et les origines de la modernité, Paris, La Fabrique, 2004.

9 AMONTONS Guillaume, « Moyen de substituer commodément l'action du feu à la force des hommes et des chevaux pour mouvoir les machines ", Histoire et mémoires de l'Académie royale des sciences, année 1699, Paris, chez G. Martin, J-B. Coignard et H.L. Guérin, 1732.

10 Il s'agissait de savoir si la bonne mesure dynamique était le produit de la masse par la vitesse (« quantité de mouvement »: $\mathrm{mV}$ ) ou de la masse par le carré de la vitesse («force vive»: $\mathrm{mv}^{2}$ ). Le travail mécanique (produit d'une force par le déplacement de son point d'application : f.d) a les mêmes dimensions physiques que la force vive. La théorie du travail a conduit à la définition du concept moderne d' " énergie cinétique », qui est égal à la demie force vive : $1 / 2 \mathrm{mv}^{2}$. 
${ }^{11}$ En fait, on ne trouve pas cette formule, pourtant régulièrement citée, chez Montgolfier lui-même, mais simplement une expression plus plate: « la force a un prix » (« Mémoire sur la possibilité de substituer le bélier hydraulique à l'ancienne machine de Marly ", Journal de l'Ecole polytechnique, 1808, p. 289-317: 290).

${ }^{12} \mathrm{Si}$ la formule de Montgolfier incarne bien cette articulation, on en trouve des traces plus anciennes, notamment chez Leibniz, qui, parlant de la dispersion de l'énergie, explique, dans sa correspondance avec Clarke, que c'est comme «changer la grosse monnaie en petite » Correspondance Leibniz-Clarke, Paris, Puf, 1957, p. 168.

${ }^{13}$ COURNOT Augustin « De la mesure des forces et du travail des machines ", dans LARDNER D. et KATER, Eléments de mécanique, trad. par A. Cournot, Paris, Paulin, 1834, repris dans VATIN François, Economie politique et économie naturelle chez A.A. Cournot, Paris, Puf, 1998, p. 375-397: 383.

${ }^{14}$ CORIOLIS Gaspard-Gustave, Du calcul de l'effet des machines, ou Considérations sur l'emploi des moteurs et sur leur évaluation, Paris, Carilian-Goeury, 1829, p. 32

15 GUILLERME Jacques et SEBESTIK Jan, «Les commencements de la technologie », Thalès, tome 12, 1968. Réédité dans Documents pour l'histoire des techniques, nouvelle série, $\mathrm{n}^{\circ} 14$, $2^{\mathrm{e}}$ semestre 2007.

${ }^{16}$ WEBER Max, Économie et société, Paris, Plon, 1971, tome 1, p. 104-105.

7 LESOURNE Jacques, Le Calcul économique, Paris, Dunod, 1964, rééd. 1972, p. 5.

${ }^{18}$ GRALL Bernard, Économie de forces et production d'utilités. L'émergence du calcul économique chez les ingénieurs des Ponts et Chaussées (1831-1891), Rennes, PUR, 2003 (manuscrit révisé et commenté par François Vatin).

19 HEUZÉ Gustave Du lait et de ses emplois en Bretagne, Nantes, 1845.

20 LE BIANIC Thomas et VATIN François, «Armand Imbert (1850-1922), la science du travail et la paix sociale », Travail et Emploi $\mathrm{n}^{\circ}$ 111, juillet-septembre 2007, p. 7-19.

${ }^{21}$ DUPIN Charles, Géométrie et mécanique des arts et métiers et des beaux-arts, Paris, Bachelier, 1826, tome 2 (cité d'après la deuxième édition, 1842, p. 82-83).

${ }^{22}$ MAUSS Marcel, «Les techniques du corps » (1935), in Techniques, technologie et civilisation, Paris, PUF, 2012, p. 365-394. 